\title{
Performance, metabolic and hormonal responses on the peripartum of grazing beef cows supplemented during the pre-partum
}

\section{CURRENT STATUS: ACCEPTED}

BMC Veterinary Research $\triangle$ BMC series

Matheus Fellipe de Lana Ferreira

Universidade Federal de Viçosa

matheus.fellipe234@gmail.comCorresponding Author

ORCiD: https://orcid.org/0000-0002-7524-8233

Luciana Navajas Rennó

Universidade Federal de Viçosa

Edenio Detmann

Universidade Federal de Viçosa

Mário Fonseca Paulino

Universidade Federal de Viçosa

Sebastião de Campos Valadares Filho

Universidade Federal de Viçosa

Samira Silveira Moreira

Universidade Federal de Viçosa

Hudson Caio Martins

Universidade Federal de Viçosa

Bruno Inácio Correa de Oliveira

Pontificia Universidade Catolica do Parana

Julia Avansi Marquez

Universidade de Sao Paulo

Isabela de Paula Cidrine

Universidade Federal de Viçosa

\section{DOI:}


10.21203/rs.2.17836/v1

\section{SUBJECT AREAS}

Small Animal Medicine

KEYWORDS

nutrition, metabolism, parturition, Zebu 
Abstract

Background: Strategies for feeding supplements during the pre-partum are usually adopted because nutritional status upon calving is the main factor to affect reproductive performance. Metabolic parameters that relate nutritional status to physiological processes can be used to better understand the effects of supplementation. This study evaluated the effects of 60-day pre-partum energy-protein supplementation on performance, metabolic and hormonal responses during the peripartum of grazing beef cows. Thirty-eight pregnant multiparous Nellore cows were assigned to a completely randomized design with two treatments: control with no supplement and supplementation on the last 60 gestation days $(1.5 \mathrm{~kg} / \mathrm{d})$.

Results: The supplemented cows had higher ADG during the pre-partum $(P<0.10)$, but ADG did not differ between treatments during the postpartum. Nonsupplemented cows' ADG did not change during these periods $(P>0.10)$. Supplementation did not affect $(P>0.10)$ BCS and calves' BW upon calving on days 45 and 90 , milk yield and composition $(P>0.10)$. No differences $(P>0.10)$ were found for forage intake and neutral detergent fiber digestibility. The intake and digestibility of CP and OM increased $(P<0.10)$ with supplementation. An interaction occurred $(P>0.10)$ between supplementation and peripartum days for BUN, $\beta \mathrm{HB}, \mathrm{T} 3$ and T4. Concentration of others blood parameters significantly changed $(P<0.10)$ along peripartum days. There was no difference in pregnancy rates and days from calving to conception among treatments $(P>0.10)$.

Conclusions: Providing energy-protein supplement for grazing Nellore cows on the last $60 \mathrm{~d}$ of gestation improve energy balance during pre-partum, however, no carryover effects are seen for prepartum supplementation on post-partum physiological responses. Keywords: nutrition, metabolism, parturition, Zebu

Full-text

Due to technical limitations, full-text HTML conversion of this manuscript could not be completed. However, the manuscript can be downloaded and accessed as a PDF. Tables 
Table 1. Ingredients and composition of supplement provided to cows at 60-days pre-partum

Item $^{1}$

Ingredients (\%; as-fed basis)

Corn meal

Soybean meal

Wheat meal

Urea:ammonium sulfate (9:1)
41.2

36.0

20.0

2.80

965

320

143

apNDF

${ }^{1} \mathrm{OM}$ - organic matter; CP - crude protein; apNDF - neutral detergent fiber corrected for ash and protein residue. Mineral mix - CaHPO4=50.00\%; $\mathrm{NaCl}=47.775 \% ; \mathrm{ZnSO}=1.4 \% ; \mathrm{Cu} 2 \mathrm{SO} 4=0.70 \% ; \mathrm{CoSO}=0.05 \% ; \mathrm{KIO}=0.05 \%$ and $\mathrm{Mr}$ $0.025 \%$.

Table 2. Uruchloa decumbes chemical composition

\begin{tabular}{lcccc}
\hline & Item & & Months \\
\cline { 2 - 5 } & August & September & October & November \\
\hline DM $^{1}$ & 651.3 & 762.3 & 505.8 & 236.7 \\
OM $^{2}$ & 931.8 & 937.6 & 934.1 & 910.4 \\
CP2 $^{2}$ & 48.8 & 52.3 & 58.3 & 82.1 \\
apNDF $^{2}$ & 749.3 & 770.1 & 731.2 & 592.5 \\
iNDF $^{2}$ & 338.7 & 347.6 & 362.1 & 177.8 \\
NDIN $^{3}$ & 217.1 & 190.4 & 259.2 & 425.6 \\
\hline
\end{tabular}

Dry matter $(D M)$, organic matter $(O M)$, crude protein $(C P)$, neutral detergent fibre corrected for ash and protein (apNDF), indigestible neutral detergent fiber (iNDF), insoluble neutral detergent nitrogen (NDIN).

$1 / \mathrm{g} / \mathrm{kg}$ of natural matter

$2 / \mathrm{g} / \mathrm{kg} \mathrm{DM}$

$3 / \mathrm{g} / \mathrm{kg}$ total nitrogen

4 / intake and digestibility assay 
Table 3. Least square means and P-values for effect of energy-protein supplementation on cows and calves' performanct

\begin{tabular}{|c|c|c|c|}
\hline \multirow[t]{2}{*}{ Item $^{1}$} & \multicolumn{2}{|c|}{ Treatment } & \multirow[t]{2}{*}{ SEM } \\
\hline & No supplementation & Supplementation & \\
\hline Calving BW $(\mathrm{kg})$ & 515.1 & 536.9 & 13.86 \\
\hline Calving BCS & 5.19 & 5.53 & 0.222 \\
\hline BCS 45 & 5.38 & 5.11 & 0.273 \\
\hline CBW (kg) & 30.8 & 32.1 & 1.14 \\
\hline CBW45 (kg) & 70.6 & 73.0 & 1.78 \\
\hline CBW90 (kg) & 93,2 & 101.1 & 6.30 \\
\hline $\begin{array}{l}\mathrm{N}^{\circ} \text { days to } \\
\text { conception }\end{array}$ & 80 & 77 & 11.30 \\
\hline $\begin{array}{l}\text { Pregnancy rate } \\
(\%)\end{array}$ & 73 & 68 & - \\
\hline
\end{tabular}

Table 4. Least square means and P-values for effect of energy-protein supplementation on milk production and composition

\begin{tabular}{cccc}
\hline Item $^{1}$ & SEM & Treatment & P-value \\
\cline { 2 - 3 } & NS & SS &
\end{tabular}




\begin{tabular}{lccll}
\hline Milk 30 & 7.69 & 7.84 & 0.432 & 0.817 \\
FCM $^{\mathrm{a}} 30$ & 8.68 & 8.43 & 0.521 & 0.742 \\
Fat (\%) & 5.00 & 4.90 & 0.205 & 0.755 \\
Protein (\%) & 2.99 & 3.02 & 0.054 & 0.697 \\
Lactose (\%) & 4.60 & 4.65 & 0.066 & 0.557 \\
Total solids (\%) & 13.40 & 13.31 & 0.285 & 0.825 \\
\hline Milk 45 & 7.74 & 8.15 & 0.424 & 0.525 \\
FCM ${ }^{\text {a }} 45$ & 9.17 & 9.41 & 0.449 & 0.717 \\
Fat (\%) & 5.40 & 5.17 & 0.244 & 0.514 \\
Protein (\%) & 3.05 & 3.09 & 0.057 & 0.598 \\
Lactose (\%) & 4.63 & 4.65 & 0.047 & 0.673 \\
Total solids (\%) & 13.89 & 14.07 & 0.221 & 0.556 \\
\hline
\end{tabular}

${ }^{\mathrm{a}} \mathrm{FMC}=4 \%$ fat-corrected milk yield ( 30 and 45 days)

Table 5. Least square means and P-values for effect of energy-protein supplementation on cow's intake during pre-calvir

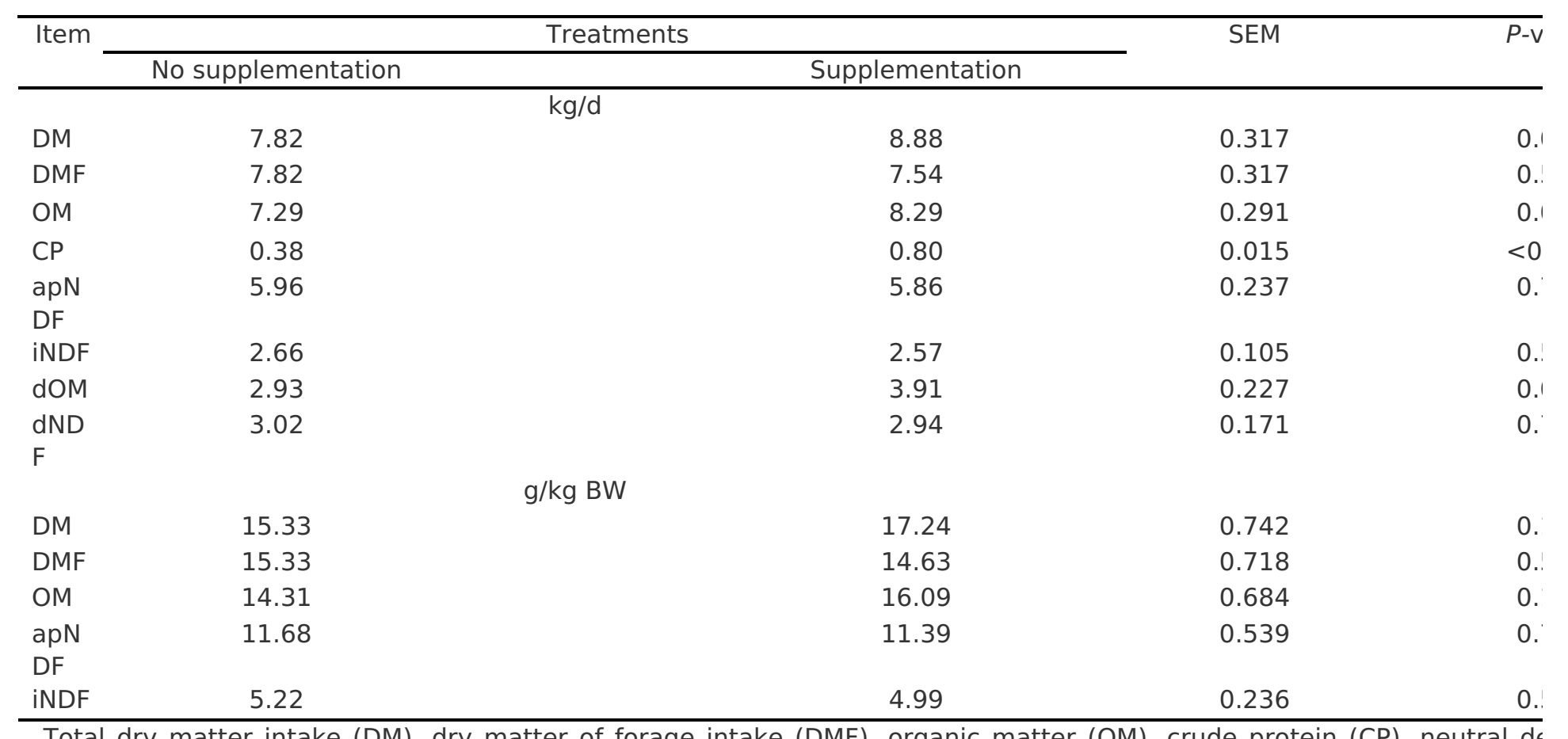

Total dry matter intake (DM), dry matter of forage intake (DMF), organic matter (OM), crude protein (CP), neutral df corrected for ash and protein (apNDF), indigestible NDF (iNDF), digested organic matter (dOM), disgested NDF (dNDF). 
Table 6. Least square means and P-values for effect of energey-protein supplementation on apparent digestibility and synthesis of nitrogen compounds during pre-calving

\begin{tabular}{lcccc}
\hline \multirow{2}{*}{ Item } & \multicolumn{2}{c}{ Treatments } & & $P$-value \\
\cline { 2 - 5 } & No supplementation & Supplementation & SEM & Sup \\
\hline OM & 39.86 & 47.32 & 1.522 & 0.013 \\
CP & 2.77 & 50.65 & 1.623 & $<0.001$ \\
apNDF & 50.67 & 50.21 & 0.014 & 0.832 \\
Nmic & 89.24 & 92.69 & 66.535 & 0.370 \\
Emic & 190.06 & 148.16 & 12.666 & 0.036 \\
\hline
\end{tabular}

Organic matter (OM, \%), crude protein (CP, \%), neutral detergent fiber corrected for ash and protein (apNDF, \%), digestec organic matter (dOM, g/kg DM), ruminal synthesis of microbial nitrogen (NMic, g/d), efficiency for synthesis of microbial pI (Emic, g microbial CP synthesis kg dOM intake). 
Table 7. Least square means and P-values for effect of supplementation on serum metabolites and hormones during pre

\begin{tabular}{|c|c|c|c|}
\hline \multirow[t]{2}{*}{ Item } & \multicolumn{2}{|c|}{ Treatments } & \multirow[b]{2}{*}{ SEM } \\
\hline & No supplementation & Supplementation & \\
\hline Glucose, mg/dL & 62.97 & 63.70 & 1.895 \\
\hline Triglycerides, mg/dL & 26.74 & 27.07 & 1.085 \\
\hline Total cholesterol, mg/dL & 132.04 & 142.47 & 5.385 \\
\hline VLDL mg/ dL, & 5.34 & 5.41 & 5.385 \\
\hline LDL mg/ dL, & 56.21 & 66.15 & 5.054 \\
\hline HDL mg/ dL, & 69.45 & 70.58 & 3.339 \\
\hline Creatinine, mg/dL & 1.40 & 1.39 & 0.054 \\
\hline BUN, mg/dL & 14.46 & 15.18 & 0.836 \\
\hline Total Proteins, g/dL & 7.39 & 7.43 & 0.124 \\
\hline Albumin, g/dL & 3.26 & 3.24 & 0.042 \\
\hline Globulins, g/dL & 4.15 & 4.19 & 0.149 \\
\hline $\mathrm{NEFA}, \mathrm{mmol} / \mathrm{L}^{1}$ & 0.33 & 0.27 & 0.042 \\
\hline$\beta \mathrm{HB}, \mathrm{mmol} / \mathrm{L}^{1}$ & 0.47 & 0.45 & 0.021 \\
\hline IGF-1, ng/dL & 184.64 & 196.54 & 16.670 \\
\hline Insulin, $\mu \mathrm{IU} / \mathrm{mL}$ & 2.99 & 2.83 & 0.324 \\
\hline $\mathrm{T} 3, \mathrm{ng} / \mathrm{mL}$ & 0.637 & 0.823 & 0.1305 \\
\hline $\mathrm{T} 4, \mu \mathrm{g} / \mathrm{dL}$ & 4.66 & 5.81 & 0.689 \\
\hline Progesterone, ng/mL, & 0.87 & 1.02 & 0.2461 \\
\hline
\end{tabular}

1/Non-esterified fatty acids (NEFA); $\beta$-hydroxybutyrate ( $\beta \mathrm{HB}$ )

2/ Supplementation (Sup)

3/ Day relative to calving (Day)

\section{Figures}




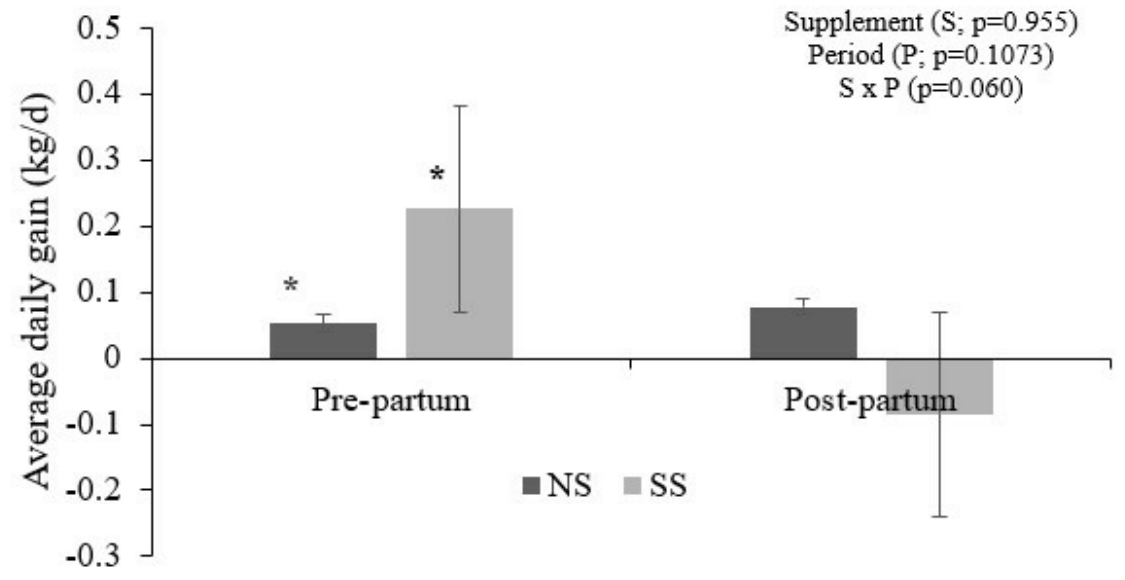

Figure 1

Average daily gain during pre- and post-partum period. Asterisks (*) indicate significant differences between treatments $(\mathrm{P}<0.10)$. NS: Nonsupplemented cows, SS: Supplemented cows. 


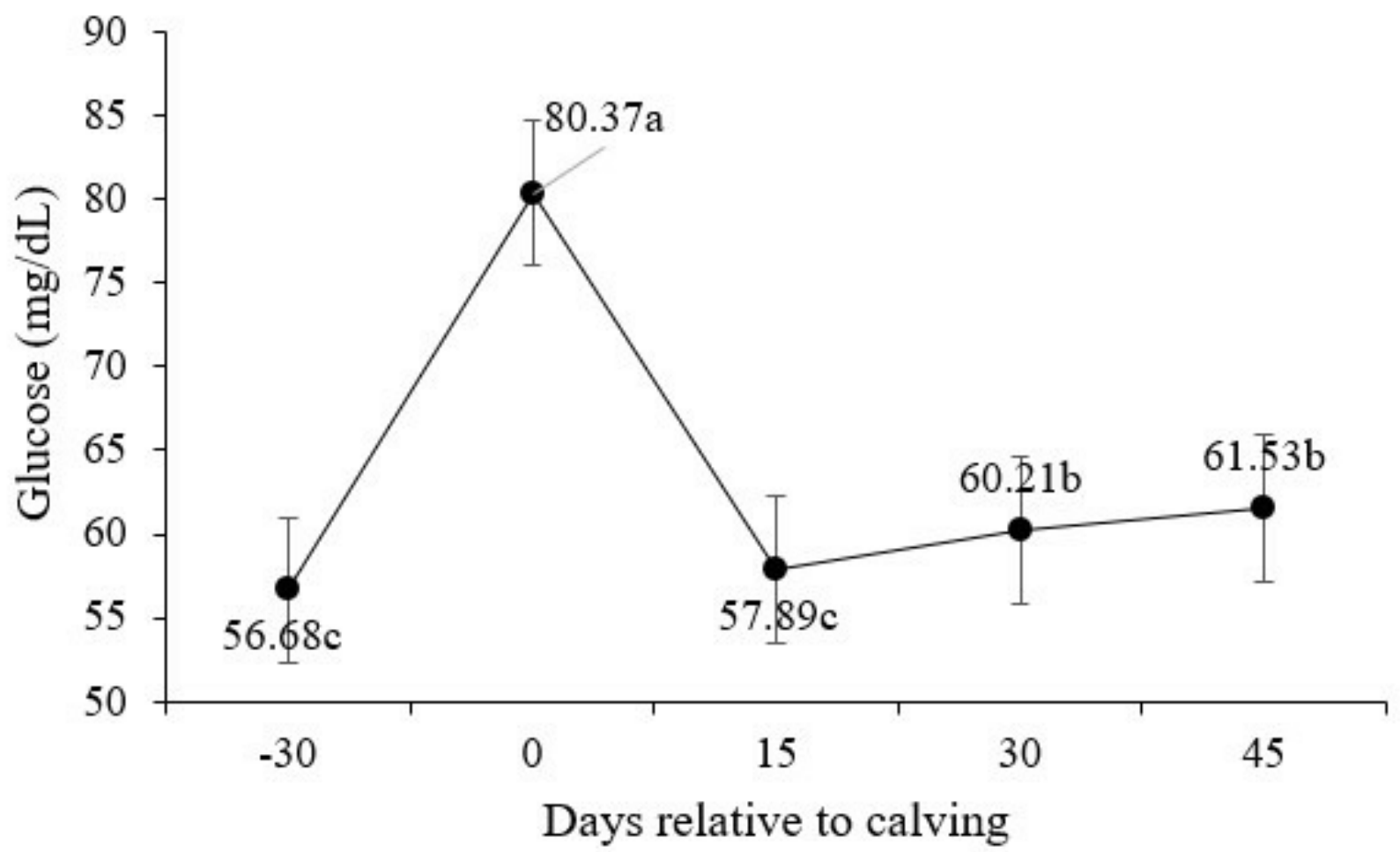

Figure 2

Glucose plasma concentrations during pre- and post-calving. Different letters indicate significant differences between days $(P<0.10)$. 


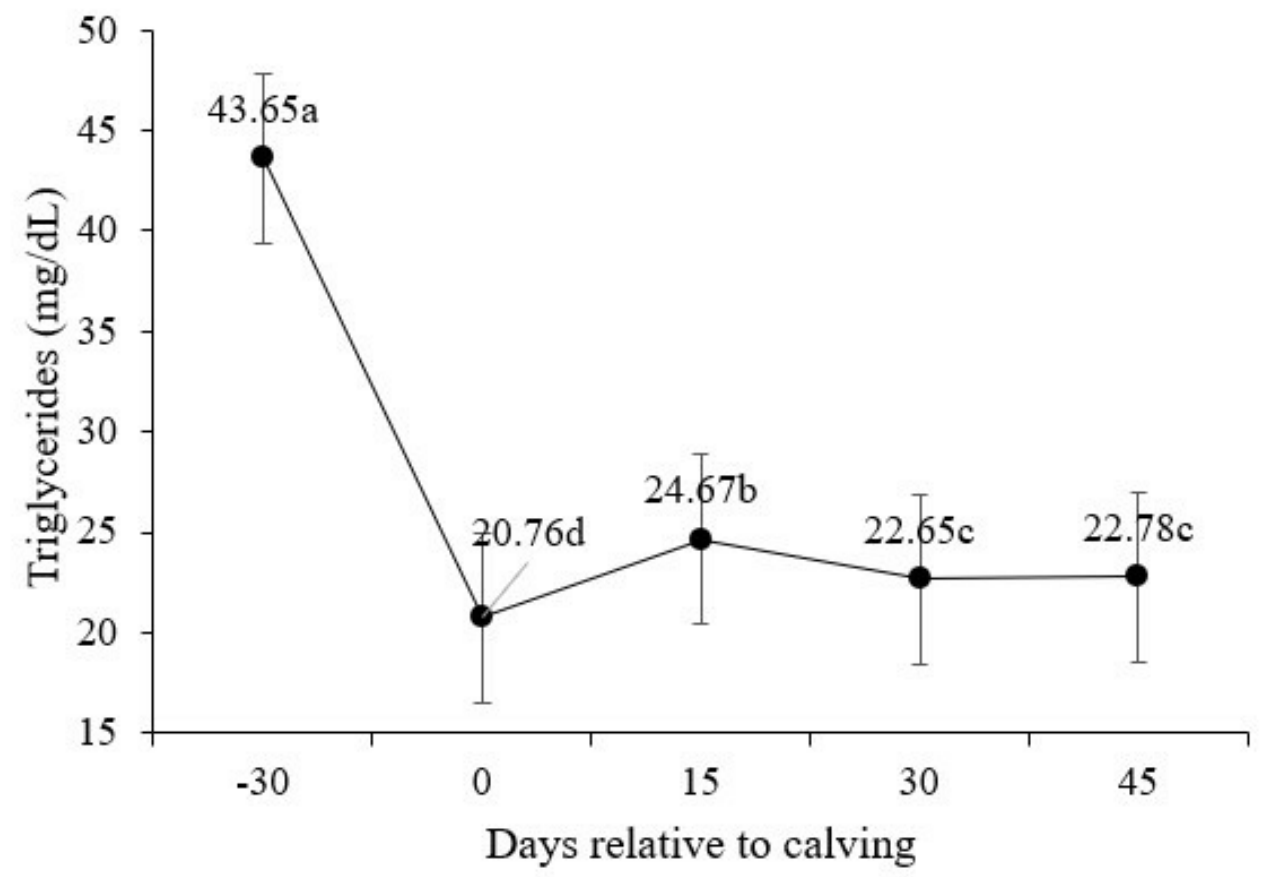

Figure 3

Triglycerides serum concentrations during pre- and post-calving. Different letters indicate significant differences between days $(P<0.10)$.

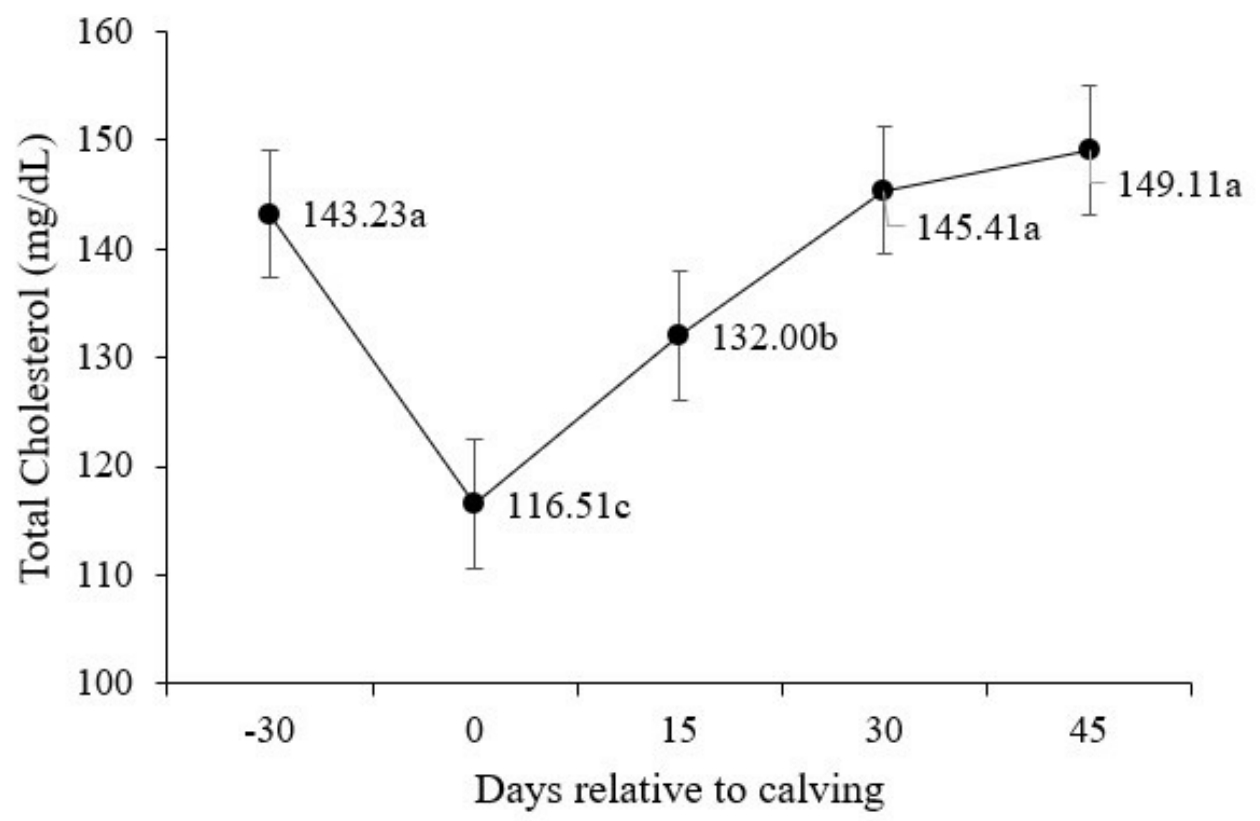

Figure 4

Total cholesterol serum concentrations during pre- and post-calving. Different letters indicate significant differences between days $(P<0.10)$. 
(a)

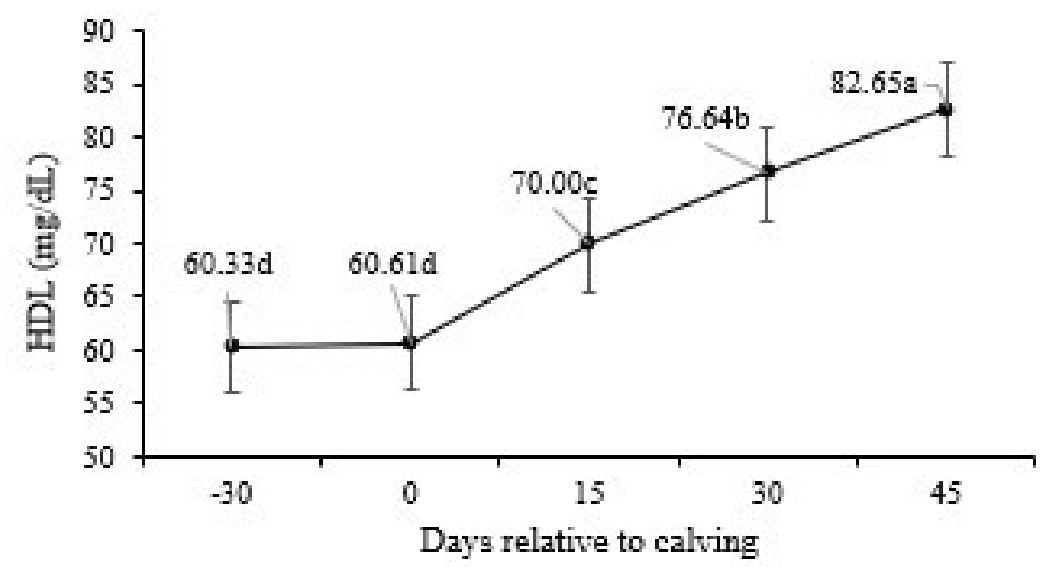

(b)

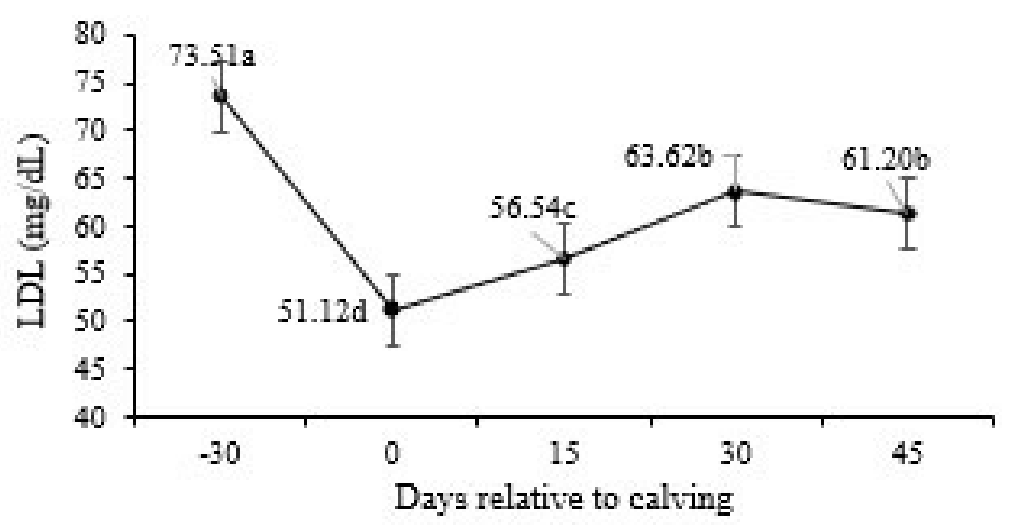

(c)

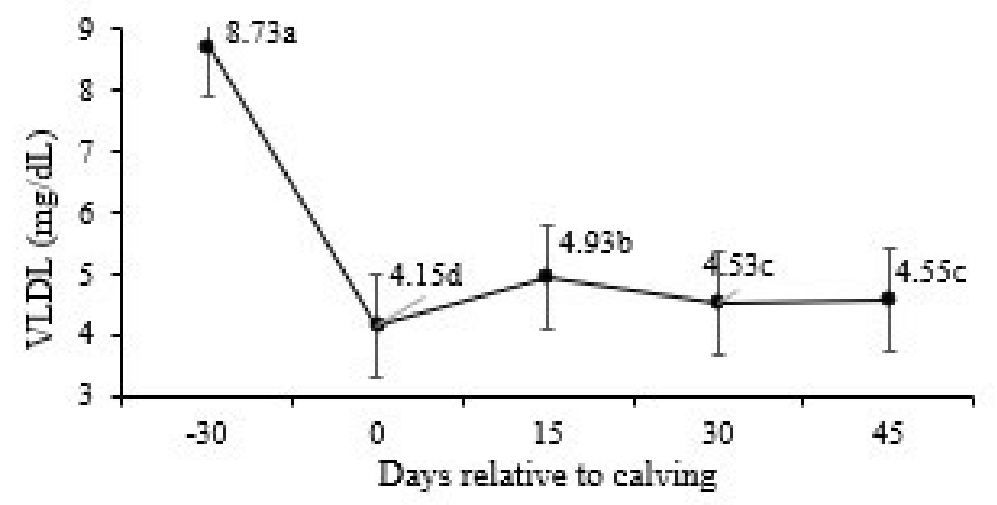

Figure 5

HDL (a), LDL (b) and VLDL (c) serum concentrations during pre- and post-calving. Different letters indicate significant differences between days $(P<0.10)$. 
(a)
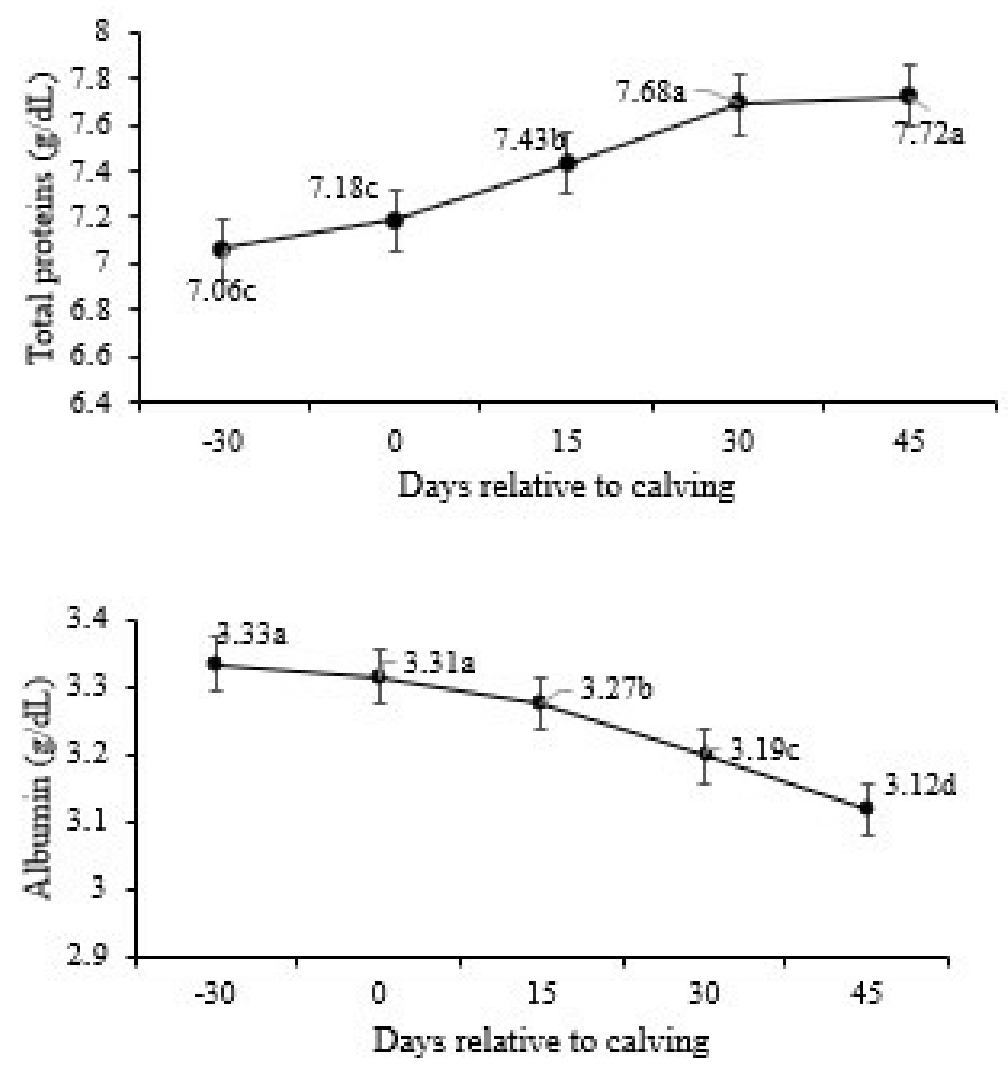

(c)

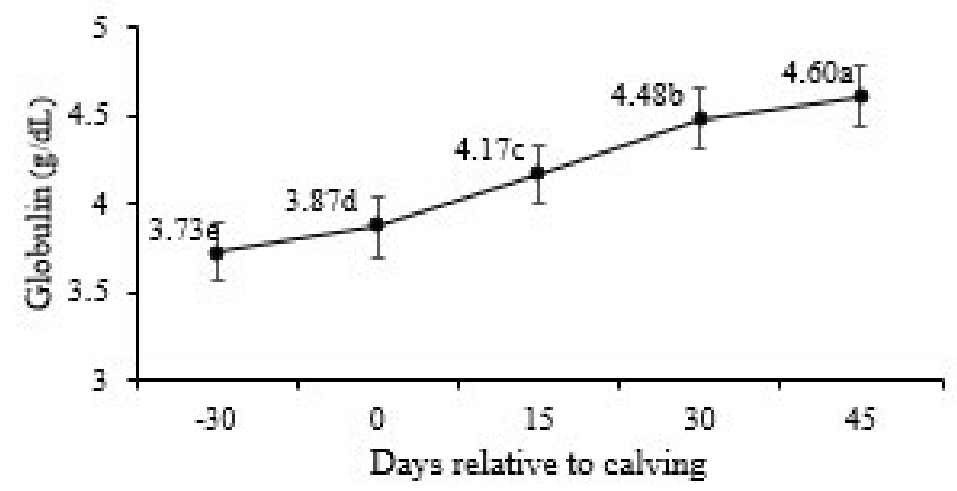

Figure 6

Total protein (a), albumin (b) and globulin (c) serum concentrations during pre- and postcalving. Different letters indicate significant differences between days $(P<0.10)$. 


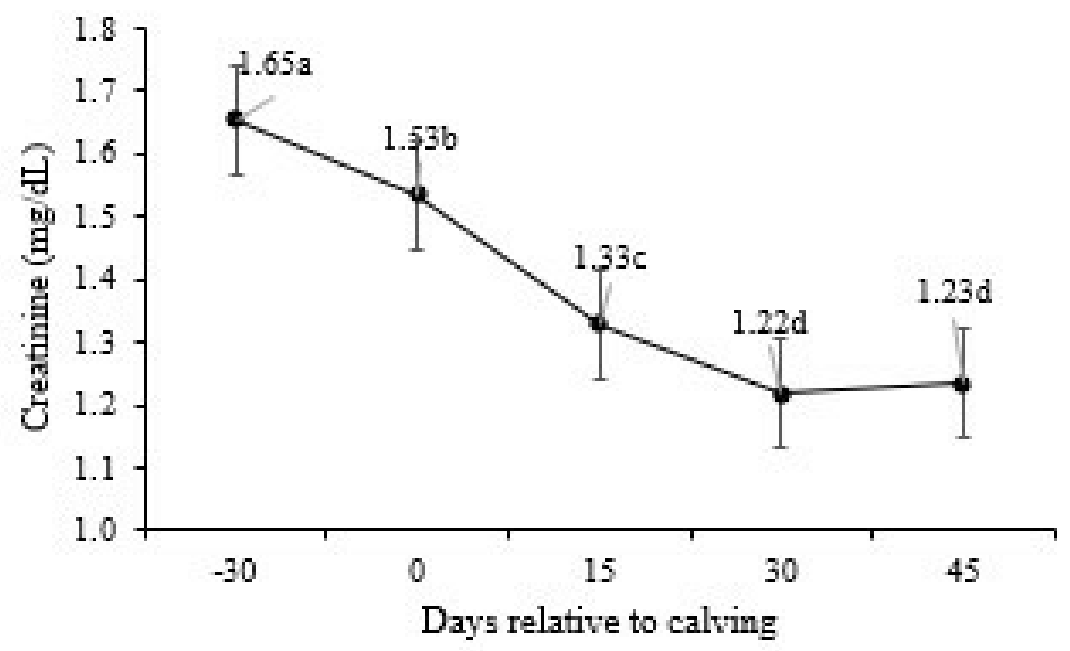

Figure 7

Creatinine serum concentrations during pre- and post-calving. Different letters indicate significant differences between days $(P<0.10)$.

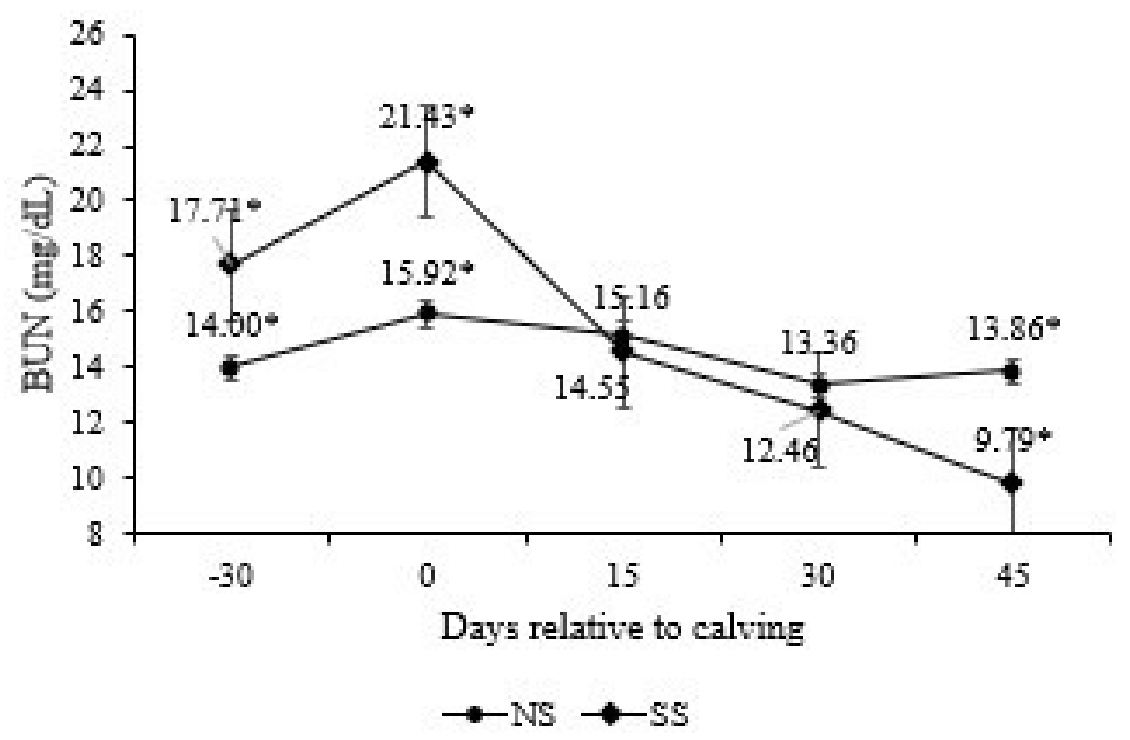

Figure 8

Blood urea nitrogen (BUN) concentrations during pre- and post-calving. Numbers followed by asterisks $(*)$ are significantly different between treatments $(P<0.10)$. 


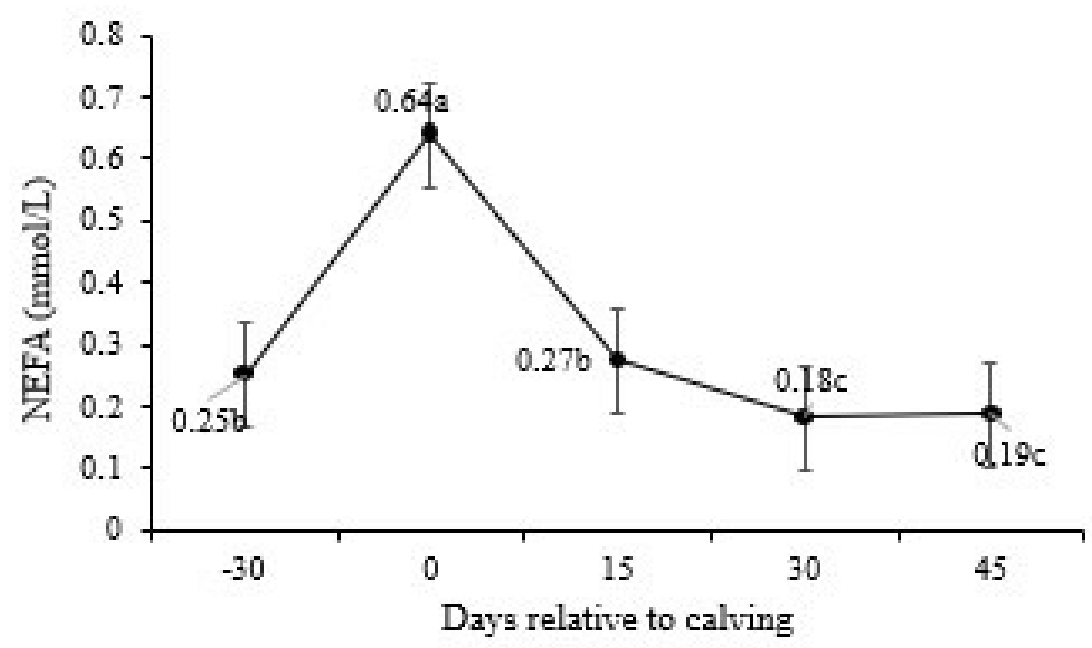

Figure 9

Non-esterified fatty acids (NEFA) serum concentrations during pre- and post-calving.

Different letters indicate significant differences between days $(P<0.10)$.

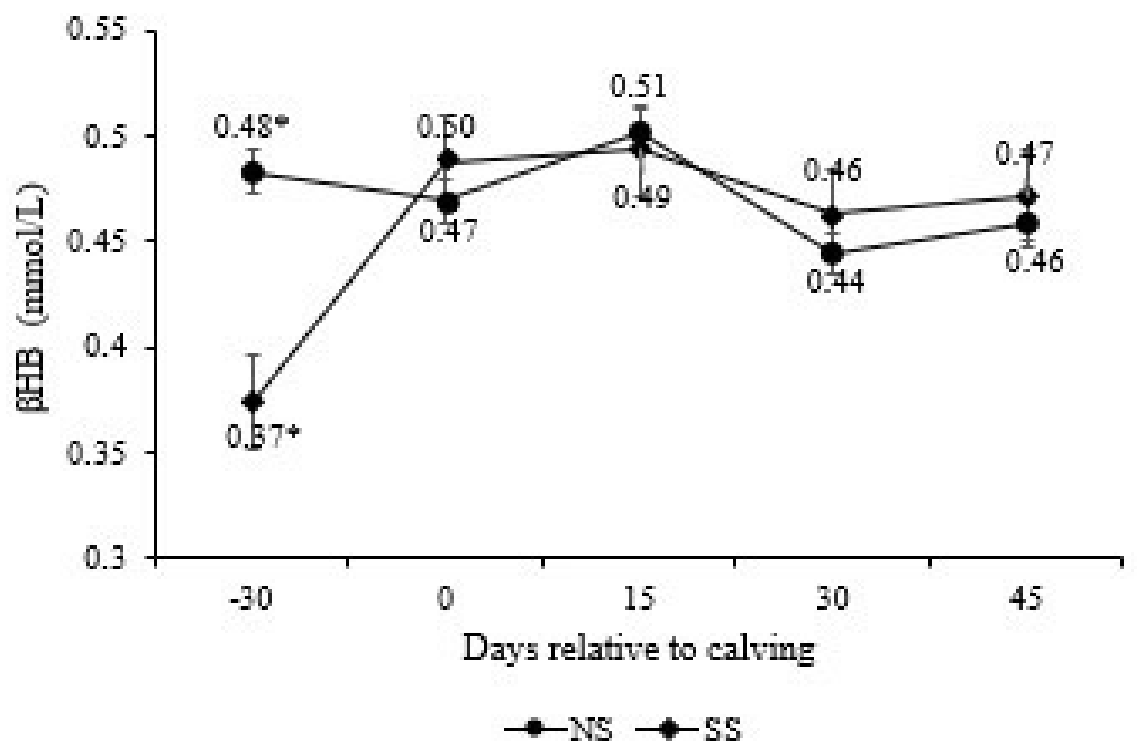

Figure 10

$\beta$-hydroxybutyrate $(\mathrm{BHB})$ serum concentrations during pre- and post-calving. Numbers followed by asterisks $(*)$ are significantly different between treatments $(P<0.10)$. 
(a)

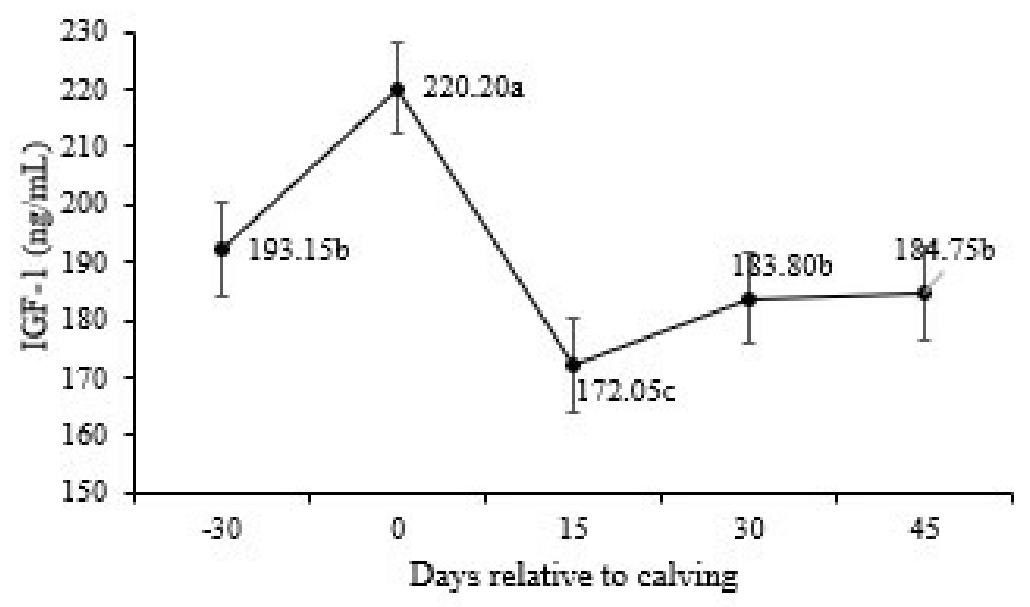

(b)

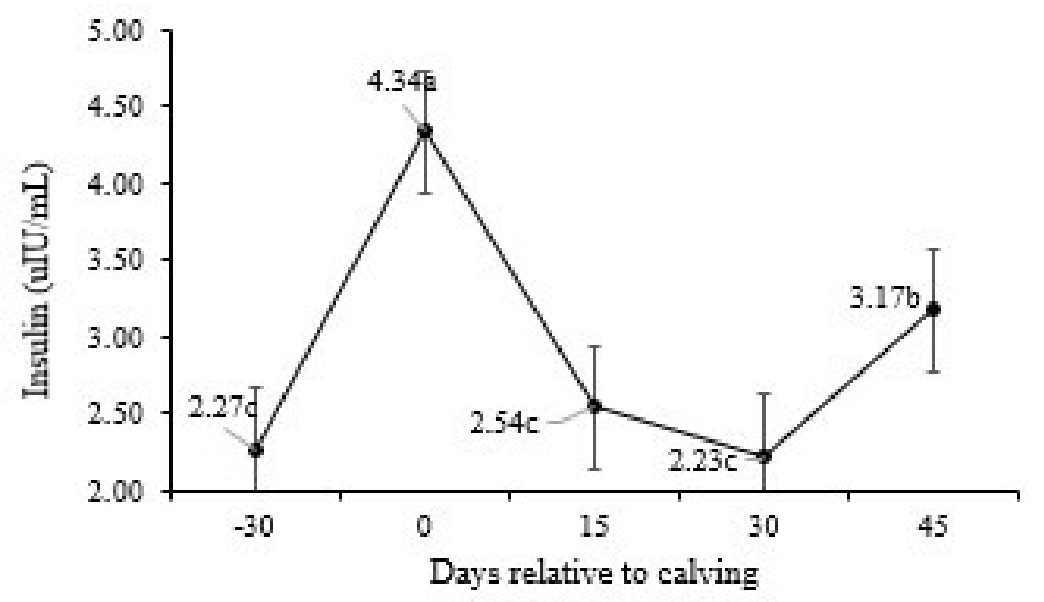

Figure 11

IGF-1 (a) e Insulin (b) serum concentrations during pre- and post-calving. Different letters indicate significant differences between days $(P<0.10)$. 
(a)
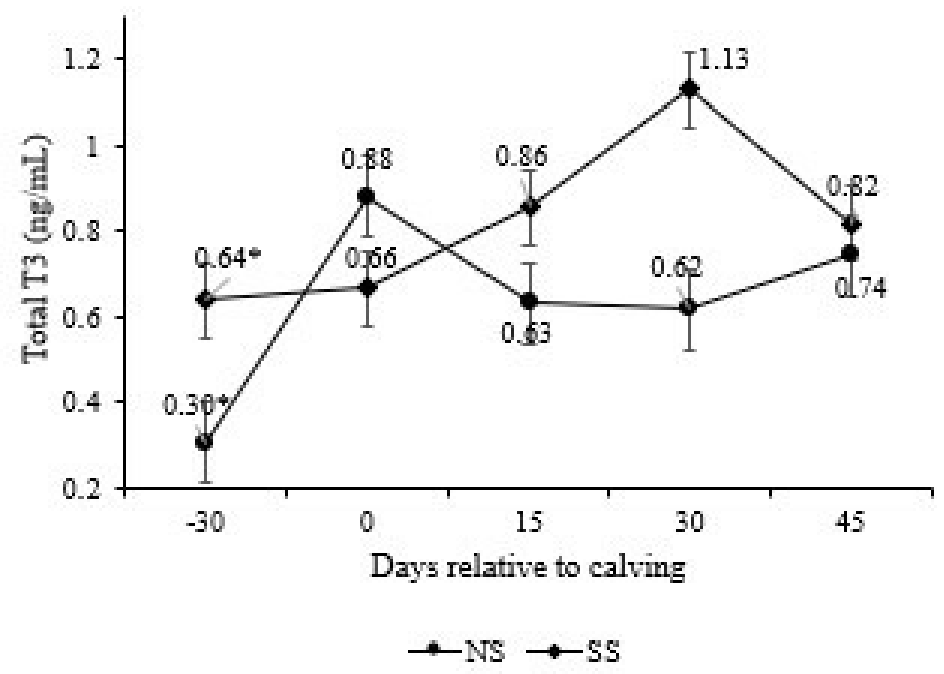

(b)

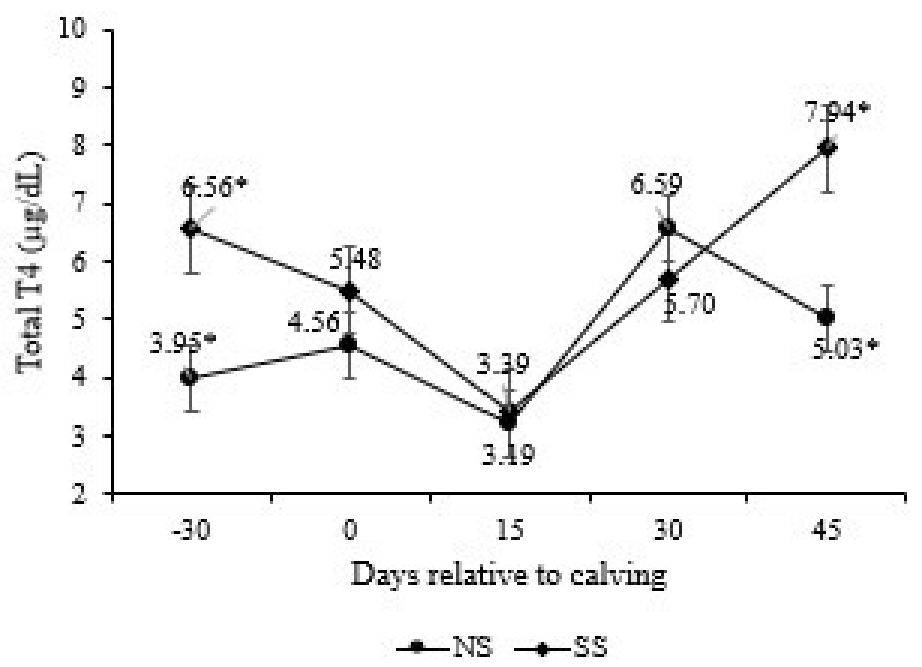

Figure 12

Total T3 (a) and T4 (b) serum concentrations during pre- and post-calving. Numbers followed by asterisks $(*)$ are significantly different between treatments $(P<0.10)$. 